\title{
Identification, cloning, and functional expression of three glutathione transferase genes from Aspergillus fumigatus
}

\author{
Claire Burns ${ }^{1}$, Rachel Geraghty ${ }^{1}$, Claire Neville, Alan Murphy, \\ Kevin Kavanagh, Sean Doyle*
}

National Institute for Cellular Biotechnology, Department of Biology, National University of Ireland, Maynooth, Co. Kildare, Ireland

Received 13 October 2004; accepted 3 January 2005

\begin{abstract}
Analysis of the genome of the human pathogen, Aspergillus fumigatus, revealed the presence of several putative glutathione transferase (GST) open reading frames. Three $A$. fumigatus GST genes, termed gst $A, B$, and $C$, were cloned and recombinant proteins expressed in Escherichia coli. Functional analysis of recombinant gstA-C confirms that the enzymes exhibit GST activity and glutathione peroxidase activity. RT-PCR confirmed low basal expression of $g$ st $A$ and $g s t C$ which was markedly up-regulated (at least $4 \times-$ $10 \times$ ) in the presence of either $\mathrm{H}_{2} \mathrm{O}_{2}$ or 1-chloro-2,4-dinitrobenzene (CDNB). Gst $B$ expression was only observed in the presence of CDNB. These results demonstrate for the first time the existence of three functional GSTs in A. fumigatus and strongly suggest a role for these enzymes in the response of the organism to both oxidative stress and xenobiotic presence.
\end{abstract}

(c) 2005 Elsevier Inc. All rights reserved.

Keywords: GST; MALDI-TOF; Xenobiotic resistance; Oxidative stress; Fungal

\section{Introduction}

Aspergillus fumigatus is a human pathogenic fungus capable of inducing a range of disease states in patients with pre-existing lung damage or immunosuppression following organ transplantation (Daly and Kavanagh, 2001). Three forms of aspergillosis are recognised clinically: saprophytic, allergic, and invasive, with the latter form having a mortality rate of $>90 \%$ in some patient groups (Denning, 1998). Conventional therapy relies upon the use of amphotericin B and, more recently, on novel azole derivatives and the echinocandin class of anti-fungal agents, but mortality rates remain high. $A$. fumigatus displays the ability to withstand attack by macrophages and neutrophils and develop in a potentially hostile environment. Toxin-mediated inhibition of

\footnotetext{
* Corresponding author. Fax: +3531 1083845 .

E-mail address: sean.doyle@nuim.ie (S. Doyle).

${ }^{1}$ These authors contributed equally to this work.
}

oxidative burst in alveolar macrophages and polymorphonuclear leukocytes by conidia and hyphae is well characterised (Bertout et al., 2002; Mitchell et al., 1997; Muryama et al., 1996). In addition, the physical size of developing hyphae prevent phagocytosis by alveolar macrophages and there is emerging evidence that A. fumigatus may be able to tolerate entry of xenobiotics as a result of amphotericin B treatment creating apertures in the fungal cell membrane (Ellis, 2002).

Glutathione transferases (GST; EC 2.5.1.18) are dimeric phase II detoxification enzymes with the ability to conjugate a broad range of potentially harmful xenobiotics to glutathione (GSH), thereby rendering them more susceptible to removal from the cell. GSTs have also been shown to exhibit GSH-dependent peroxidase activity and thus may be involved in resistance to oxidative stress. Cytosolic GSTs have been identified in almost all organisms, with mammalian GSTs the most clearly characterised. These enzymes have been implicated in pesticide resistance in plants and insects (Sheehan et al., 2001), and 
some GST polymorphisms are thought to alter cancer susceptibility in mammals (Hayes and Pulford, 1995). GSTs are divided into several classes based upon substrate specificity, sequence similarity (particularly in the $\mathrm{N}$-terminal region which is involved in GSH binding), immunological cross-reactivity and, where available, structure similarity. GST classes include $\alpha, \mu, \pi, \theta, \sigma, \zeta, \omega$, and $\kappa$ classes, with insect specific ( $\delta$ and $\epsilon$ ), plant specific ( $\varphi$ and $\tau$ ) and bacterial $(\beta)$ classes also described (Sheehan et al., 2001). In addition, it is likely that many more classes have been already characterised exist in the broad ranging GST category; for example, new protozoan and fungal GST classes have been proposed (Cha et al., 2001; Takada et al., 2004).

Until recently, relatively little was known about the presence and role of GST in fungi, however it is now clear that GST isoforms exist in a number of fungal species including Schizosaccharomyces pombe, Aspergillus nidulans, Saccharomyces cerevisiae, Issatchenkia orientalis, Yarrowia lipolytica, Cunninghamella elegans, Mucor circinelloides, and Phanerochaete chrysosporium (Cha et al., 2001; Choi et al., 1998, 2002; Dowd et al., 1997; Dowd and Sheehan, 1999; Foley and Sheehan, 1998; Fraser et al., 2002; Kim et al., 2001; Tamaki et al., 1999; Shin et al., 2002; Veal et al., 2002). Fungal GSTs exhibit differential expression patterns, with some isoforms shown to be expressed inducibly in the presence of xenobiotics or oxidative stress. For example, of two GSTs identified in I. orientalis, only one was constitutively expressed, and both were induced in the presence of $o$-dinitrobenzene $(o$-DNB) (Choi et al., 1998). Three GSTs in S. pombe were induced by oxidative stress, and mutants lacking $g s t 1^{+}$and $g s t 2^{+}$ or $g s t 3^{+}$were more sensitive to the presence of the antifungal drug fluconazole, thereby indicating a role for GST in mediating anti-fungal drug tolerance (Cho et al., 2002; Kim et al., 2001; Shin et al., 2001; Veal et al., 2002).

The identification of a functional theta class GST (gene: gst $A$ ) in $A$. nidulans has further elucidated the role of GST in fungal metabolism. Gst $A$ appears to be upregulated by the presence of either 1-chloro-2,4-dinitrobenzene $(\mathrm{CDNB})^{2}$ or $\mathrm{H}_{2} \mathrm{O}_{2}$ in the culture medium and may also play a role in mediating heavy metal resistance in A. nidulans (Fraser et al., 2002).

Given the significance of $A$. fumigatus as a human pathogen and the limited success of anti-fungal agents to treat aspergillosis, particularly in immunocompromised patients, it is surprising that the putative presence and role of GST has merited little attention. In addition, the potential role of fungal GST in allowing A. fumigatus to withstand neutrophil attack may represent a key element in the cell's ability to survive in the host and colonise

\footnotetext{
${ }^{2}$ Abbreviations used: CDNB, 1-chloro-2,4-dinitrobenzene; DCNB, 1,2-dichloro-4-nitrobenzene; GST, glutathione transferase; GSH, glutathione; MALDI-TOF, matrix assisted laser desorption ionisation-time of flight; MEM, minimal essential medium Eagle; CALM, calmodulin.
}

pulmonary tissue. Here we describe the identification, cloning, heterologous expression and characterisation of three GST genes from $A$. fumigatus. We also investigate the response of GST gene expression following exposure of $A$. fumigatus to both CDNB and $\mathrm{H}_{2} \mathrm{O}_{2}$.

\section{Experimental}

\subsection{Genomic DNA isolation}

Aspergillus fumigatus ATCC 26933 (obtained from the American Type Culture Collection, Maryland, USA) was used in this study. Aspergillus cultures were grown in $5 \%(\mathrm{v} / \mathrm{v})$ fetal calf serum in minimal essential medium Eagle (MEM) (Sigma-Aldrich, Dorset, UK) for 2 days at $37^{\circ} \mathrm{C}$. Genomic DNA was isolated as described by Nicholson et al. (2001). Briefly, ca. $4 \mathrm{~g}$ A. fumigatus mycelia were crushed in liquid $\mathrm{N}_{2}$ and suspended in $10 \mathrm{ml}$ extraction buffer $(10 \mathrm{mM}$ Tris- $\mathrm{HCl}, 10 \mathrm{mM}$ EDTA, 0.5\% (w/v) SDS pH 8.0). Phenol:chloroform:isoamyl alcohol $(25: 24: 1,10 \mathrm{ml})$ was added to the mycelial suspension and mixed gently for $30 \mathrm{~min}$. Phases were separated by centrifugation at $5000 \mathrm{~g}$ at $4^{\circ} \mathrm{C}$. The aqueous layer was removed a fresh tube and phenol extraction repeated until the interface was clear. The final aqueous layer was treated with chloroform:isoamyl alcohol (24:1) and phases separated as before. The remaining aqueous layer was treated with ribonuclease A $(20 \mu \mathrm{l}$; $10 \mathrm{mg} \mathrm{ml}^{-1}$ ) at $37^{\circ} \mathrm{C}$ for $30 \mathrm{~min}$, followed by phenol extraction, then chloroform extraction. The DNA was precipitated from the aqueous layer with 2 volumes of $100 \%$ ethanol and $1 / 10$ volume of $\mathrm{LiCl}(4 \mathrm{M})$ at $-20^{\circ} \mathrm{C}$ overnight. DNA was recovered by centrifugation at $13,000 \mathrm{~g}$ for $10 \mathrm{~min}$. The pellet was washed with $70 \%(\mathrm{v} / \mathrm{v})$ ethanol, air-dried, and resuspended in $1 \mathrm{ml}$ TE buffer (10 mM Tris-HCl, 1 mM EDTA, $\mathrm{pH} 8.0$ ).

\subsection{DNA sequence and bioinformatic analysis}

All DNA sequence analysis was performed using a Perkin-Elmer ABI Prism 310 genetic analyser, commercially by MWG Biotech (Milton Keynes, UK) or Lark Technologies (Essex, UK) and sequence similarities were determined using the BLAST algorithm (www.ncbi.nlm. nih.gov/blast/bl2seq/bl2.html). Sequence alignments and neighbor-joined phylogenetic trees were generated using ClustalW (Thompson et al., 1994; http://www.ebi.ac.uk/ clustalw). A bootstrapping value of 1000 was used, with bootstrapping percentages noted at tree branch points. Trees were visualised in Treeview (Page, 1996; http:// taxonomy.zoology.gla.ac.uk/rod/rod.html). Preliminary sequence data was also obtained from The Institute for Genomic Research website at http://www.tigr.org. Sequencing of $A$. fumigatus genome is near completion with support from the Wellcome Trust and NIH. 


\subsection{PCR amplification}

All PCR reagents were obtained from Sigma-Aldrich. PCR was performed using AccuTaq polymerase with 1$10 \mathrm{ng}$ genomic DNA as template and $1.0 \mu \mathrm{M}$ each of forward and reverse primer (Table 1) in a total volume of $50 \mu$ l. PCR conditions were as follows: $95^{\circ} \mathrm{C}$ denaturation for $5 \mathrm{~min}$; $\left(94^{\circ} \mathrm{C}\right.$ denaturation for $30 \mathrm{~s}, 55^{\circ} \mathrm{C}$ annealing for $90 \mathrm{~s}, 72^{\circ} \mathrm{C}$ extension for $\left.60 \mathrm{~s}\right) \times 35$ cycles; $68^{\circ} \mathrm{C}$ extension for $7 \mathrm{~min}$. Optimal cDNA amplification was found to require 45 cycles of PCR. PCR-amplified DNA was electrophoresed on $1 \%(w / v)$ agarose containing $0.5 \mu \mathrm{g} \mathrm{ml}^{-1}$ of ethidium bromide for $30 \mathrm{~min}$ at $100 \mathrm{~V}$. Visualisation of amplicons was performed using an 'Eagle-Eye II' digital still video system (Stratagene, CA, USA).

\subsection{Cloning and expression of gst $A, B$, and $C$}

The gst $A$ sequence was amplified from cDNA and the $g s t B / g s t C$ sequences were amplified from $A$. fumigatus DNA, using primers incorporating terminal EcoRI and Pst $\mathrm{I}$ sites to facilitate downstream cloning (Table 1). PCR products were cloned into the pCR2.1 cloning vector (Invitrogen, Carlsbad, CA, USA) according to the manufacturer's instructions. gst A, gst B, and gst $C$ were subsequently cloned into the pProEX-Hta expression vector (Invitrogen), which facilitates (His) 6 affinity tag introduction, utilising the engineered restriction sites. Ligations were performed using Quickstick ligase (Bioline, London, UK) according to the manufacturer's instructions. pPXAgst $A, \mathrm{pPXA} g s t B$, and pPX$\mathrm{A} g s t C$, the resultant expression vectors containing gst $A$, $g s t B$, and $g s t C$, respectively, were individually transformed into $E$. coli strain $\mathrm{DH} 5 \alpha$ by electroporation according to Dower et al. (1988). Expression of all three recombinant GST proteins was induced by the addition of $0.6 \mathrm{mM}$ isopropyl $\beta$-D-thiogalactoside (IPTG) and monitored by SDS-PAGE and Western blot analysis. For enzyme purification, induced cells were lysed by incubation with lysozyme $\left(90 \mu \mathrm{g} \mathrm{ml}^{-1}\right)$ and sodium deoxycholate $(0.04 \% \quad(\mathrm{w} / \mathrm{v}))$, in the presence of protease inhibitors $\left(1 \mu \mathrm{g} \mathrm{ml}^{-1}\right.$ leupeptin and pepstatin, respectively, and $1 \mathrm{mM}$ PMSF). Cell debris was removed by centrifugation at $10,000 \mathrm{~g}$ for $10 \mathrm{~min}$ and $\mathrm{N}$ terminal (His) 6 -tagged recombinant proteins were purified from the supernatant by Ni-NTA chromatography (Qiagen, West Sussex, UK) by elution with $250 \mathrm{mM}$ imidazole in $50 \mathrm{mM}$ sodium phosphate $/ 300 \mathrm{mM} \mathrm{NaCl}$. Purified GST proteins were dialysed (twice; once overnight, and once for $4 \mathrm{~h}$ ) against phosphate-buffered saline (PBS) containing $0.02 \%(\mathrm{w} / \mathrm{v})$ sodium azide for storage at $4{ }^{\circ} \mathrm{C}$. Protein concentrations were determined using the Bradford method (Bradford, 1976) with bovine serum albumin as a standard.

\subsection{MALDI-TOF MS}

Mass spectrometry was carried out using an Ettan MALDI-TOF mass spectrometer (Amersham Biosciences (Europe) GmbH, Freiburg, Germany). Protein samples for peptide mass determination were either (i) separated by SDS-PAGE and digested with trypsin or (ii) obtained following in-solution enzymatic digestion and deposited $(1 \mu \mathrm{l})$ with $1 \mu \mathrm{l} \alpha$-cyano-4-hydroxycinnaminic acid (4-HCCA; $5 \mathrm{mg} / 200 \mu \mathrm{l} 50 \%(\mathrm{v} / \mathrm{v})$ acetonitrile in aqueous trifluoroacetic acid) onto mass spectrometry slides and allowed to dry prior to delayed extraction, reflectron TOF analysis at $20 \mathrm{kV}$.

\subsection{GST activity assays}

Glutathione transferase activity was determined using methods based on those described (Habdous et al., 2002; Habig and Jakoby, 1981) whereby the change in absorbance at $340 \mathrm{~nm}\left(\Delta A_{340 \mathrm{~nm}}\right)$ was recorded, and enzyme activity calculated as micromoles CDNB utilised/mg GST/min. Activity with 1,2dichloro-4-nitrobenzene (DCNB) was performed at $345 \mathrm{~nm}$ in the same way as for CDNB, with some exceptions; $100 \mathrm{mM}$ phosphate buffer ( $\mathrm{pH} 7.5), 100 \mathrm{mM}$ DCNB in $100 \%$ ethanol, and $50 \mathrm{mM}$ GSH in phosphate

Table 1

Nucleotide sequence of oligonucleotide primers used to amplify Afugst genes $A-C$ from $A$. fumigatus genomic DNA and cDNA, respectively

\begin{tabular}{lll}
\hline Gene & Primers & Sequence $\left(5^{\prime}-3^{\prime}\right)$ \\
\hline$g s t A$ & $g s t A-\mathrm{F}$ & GAGAGAATTCATGGCAAATAGACCTGATATTACACTG \\
& $g s t A-\mathrm{R}$ & GAGACTGCAGATTAATGCTTCGCCTATTCG \\
$g s t B$ & $g s t B-\mathrm{F}$ & GAGAGAATTCATGTCTTTGAAGCCTATCGTC \\
& $g s t C-\mathrm{R}$ & GAGACTGCAGTTACTTTCCTGTGCGGC \\
& $g s t C-\mathrm{F}$ & GAGAGAATTCATGCCGGACATCCAACCCATC \\
Calmodulin & Lst $C$-R & GAGACTGCAGTCAGGTCGAGGGGAAGATGTC \\
Calmodulin & LCALM & CCGAGTACAAGGAAGCTTTCTC \\
& RCALM & GAATCATCTCGTCGACTTCGTCGTCAGT
\end{tabular}

Nucleotide sequence of control calmodulin primers (Romero et al., 2003) are also given. Oligonucleotide primers were designed based on sequence data obtained from the A. fumigatus genome sequencing effort (http://www.tigr.org). All Afugst forward primers (F1-F3) contained a 5' EcoRI restriction site, and reverse primers contained 3' PstI sites, to facilitate directional cloning into pProEx-Hta. 
buffer were used. Activity with ethacrynic acid was recorded at $270 \mathrm{~nm}$, using $100 \mathrm{mM}$ phosphate buffer, pH $6.5,20 \mathrm{mM}$ ethacrynic acid in $100 \%$ ethanol, and $2.5 \mathrm{mM}$ GSH. Glutathione peroxidase activity was determined as described (Veal et al., 2002). Briefly, test samples $(100 \mu 1)$ were mixed with $880 \mu 1$ of assay buffer (50 $\mathrm{mM}$ potassium phosphate, $\mathrm{pH} 7.0,1 \mathrm{mM}$ EDTA, $1 \mathrm{mM} \mathrm{NaN}_{3}, 0.2 \mathrm{mM} \mathrm{NADPH}, 1 \mathrm{U} \mathrm{ml}^{-1}$ glutathione reductase and $1 \mathrm{mM} \mathrm{GSH}$ ) and incubated at $30^{\circ} \mathrm{C}$ for exactly $5 \mathrm{~min}$, after which the mixture was transferred to a cuvette and $20 \mu 1$ of $69 \mathrm{mM}$ cumene hydroperoxide added. The depletion of NADPH was measured over $3 \mathrm{~min}$ at $340 \mathrm{~nm}$.

\subsection{Induction of GST expression in A. fumigatus and analysis by $R T-P C R$}

Aspergillus fumigatus ATCC 26933 was cultured, with agitation, in $500 \mathrm{ml} \mathrm{MEM}+5 \%$ (v/v) fetal calf serum at $37^{\circ} \mathrm{C}$ for $47 \mathrm{~h}$, before addition of either CDNB (final concentration: $200 \mu \mathrm{M}$ ) or $\mathrm{H}_{2} \mathrm{O}_{2}$ (final concentration: $5 \mathrm{mM})$. Aliquots $(50 \mathrm{ml})$ were removed both prior to induction and at 1,2 , and $3 \mathrm{~h}$ post-induction. $A$. fumigatus mycelia were collected by filtration, rapidly frozen in liquid nitrogen, and stored at $-80^{\circ} \mathrm{C}$ prior to RNA extraction. RNA was extracted from $A$. fumigatus mycelia using the RNeasy plant mini kit (Qiagen). Quantification of RNA was performed using Total Lab software (NonLinear Dynamics) to ensure equal amounts of RNA were used subsequently, and ca. $1 \mu \mathrm{g}$ RNA was used for cDNA synthesis. RNA was treated with DNase I (Sigma-Aldrich) prior to cDNA synthesis to remove DNA contamination. cDNA synthesis from mRNA was performed using the SuperScript kit (Invitrogen) using oligo(dT) primers. Subsequent PCR of GST cDNA was performed as described above. Control PCRs were performed with primers LCALM and RCALM (Table 1) which amplify 348 and $617 \mathrm{bp}$ regions from $A$. fumigatus cDNA and genomic DNA, respectively (Romero et al., 2003). Densitiometric quantification of PCR products was performed using Genetools software (Syngene).

\section{Nomenclature}

In accordance with recommendations from the $A$. fumigatus sequencing group (http://www.man.ac.uk), the glutathione transferase genes disclosed here are termed gst $A$, gst $B$, and $g_{s t} C$ and the corresponding proteins identified as gstA, gstB, and gstC. In addition, as recommended, the corresponding $S$. pombe orthologs are identified as superscripts as follows: gst $A^{g s t 3}$, gst $B^{g s t 1}$, and $g_{s t} C^{g s t 2}$. Finally, as recommended, the three-letter prefix 'Afu', for $A$. fumigatus gene identification, has only been used when necessary.

\section{Results and discussion}

\subsection{Cloning and sequence analysis of A. fumigatus GST open reading frames}

Similarity searching of the A. fumigatus genome database with $A$. nidulans gstA (Genbank Accession No. AAM48104; Fraser et al., 2002) revealed the presence of an $A$. fumigatus GST (AfugstA) which, following amplification with primers $g s t A-\mathrm{F} / \mathrm{R}$ yielded a PCR product of $909 \mathrm{bp}$ when a template of A. fumigatus genomic DNA was used, and 762 bp when cDNA was employed; sequence examination revealed two introns of 95 and $52 \mathrm{bp}$ proximal to the $5^{\prime}$ end. The A. fumigatus genome database (http://www.tigr.org) was also interrogated with $S$. pombe protein sequences corresponding to GSTI (Genbank Accession No. AAK77864; Cho et al., 2002) and GSTII (GenBank Accession No. AAF21054; Kim et al., 2001), and a number of GST-like open reading frames were revealed which exhibited approximately $30 \%$ sequence similarity to the query sequences. PCR primers $g s t B-\mathrm{F} / \mathrm{R}$ and $g_{s t} C-\mathrm{F} / \mathrm{R}$ (Table 1 ) were designed based on these A. fumigatus GST sequences and used to amplify open reading frames of 663 and $675 \mathrm{bp}$, respectively, from A. fumigatus genomic DNA. Gst $A, B$, and $C$ open reading frames corresponding to protein sequences shown in Fig. 1 were cloned into pProEx-Hta for recombinant expression.

Cloned gst $A, B$, and $C$ sequences were compared with the A. fumigatus genome database; observed differences were minimal, resulting in translated amino acid sequences which exhibited $100 \%$ sequence identity to the database for gstA and gstB. GstC exhibited a single amino acid difference, which was a conservative change from $\mathrm{V}$ (http://www.tigr.org) to I (gstC sequence) at position 33. GstC was independently cloned and sequenced several times, indicating a true polymorphism rather than a sequence error. From the amino acid sequence alignment of gst A to $\mathrm{C}$ shown in Fig. 1, it is apparent that isolated regions of identity exist between all three proteins throughout their entire sequence, with gstA apparently consisting of a 30 amino acid C-terminal extension relative to gstB and $\mathrm{C}$. However, more

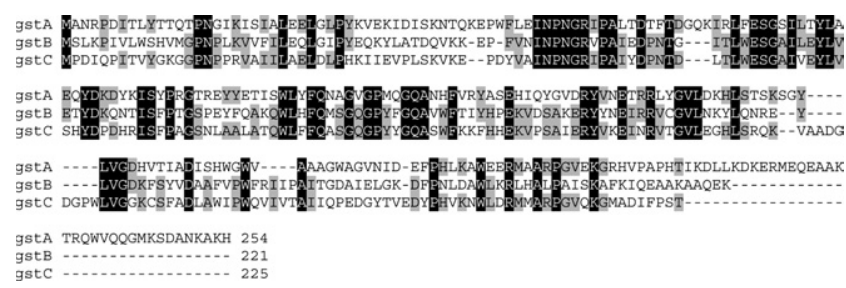

Fig. 1. Amino acid sequence alignment of and gstA (254 aa), gstB (221 aa) and gstC (225 aa). Identical residues are highlighted in black boxes while those residues only common to two sequences are shaded grey. Gaps (-) are introduced for optimal alignment. It appears that gst A contains a 30 amino acid $\mathrm{C}$-terminal extension relative to both gstB and $\mathrm{C}$. 
criteria are required to confidently assign the GSTs to this class, such as immunological cross-reactivity. As relatively few fungal GSTs have been characterised, it is difficult to determine whether these enzymes are homologues of those found in other organisms or are fungal specific. Cha et al. (2001), identified a GST from Cunninghamella elegans which bore little resemblance to known classes, and a new fungal class was proposed; it is possible that several classes are yet to be discovered.

Fig. 2 shows the relationship between GSTs and many of the fungal GST sequences characterised to date. It can be seen gstB and $\mathrm{C}$ are most closely related to the gstA enzymes from $A$. fumigatus and $A$. nidulans, respectively. While $S$. pombe GST1 and 2 also exhibit significant relatedness to the GST proteins from A. fumigatus, those from $\mathrm{I}$. orientalis and $\mathrm{Cu}$. elegans are more distantly related. When the three $A$. fumigatus GST protein sequences were used in a general BLAST search at http:/ /www.ncbi.nlm.nih.gov/entrez/, similarities to putative GST-like proteins from several fungal species, which have not been fully characterised, were revealed. GstA exhibited the highest similarities, indicating that this GST may have developed prior to speciation. Novel sequences identified using gstA included a putative GST from the artichoke pathogen Botryotinia fuckeliana (GenBank Accession No. AAG43132, 69\% identity) and putative proteins from the rice blast fungus Magnaporthe grisea (GenBank Accession No. EAA55090, 58\% identity), Neurospora crassa (GenBank Accession No. CAD36970, 58\% identity), and the cereal pathogen Gibberella zeae (GenBank Accession No. EAA71824, 49\% identity). GstB and $\mathrm{C}$ also showed similarity to the $B$. fuckeliana putative GST, at the level of $40 \%$ identity. While gstB also exhibits similarity to the $N$. crassa protein mentioned previously (GenBank Accession No. CAD36970, 44\% identity) and gstC shares similarity with the G. zeae protein ( $39 \%$ identity), there is little

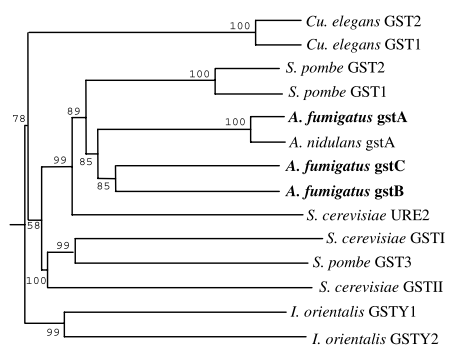

Fig. 2. Phylogenetic analysis of the three A. fumigatus GST proteins compared to 11 other characterised fungal GSTs. Sequences were aligned and a neighbor-joined tree generated using ClustalW, with bootstrapping of 1000 . Percentage bootstrapping values are shown at branch points. Sequence GenBank Accession numbers are as follows: Cunninghamella elegans GST2, AAL02369; $\mathrm{Cu}$. elegans GST1, AAL02368; $\mathrm{S}$. pombe GST2, AAF21054; $S$. pombe GST1, AAK77864; A. nidulans GSTA, AAM48104; S. cerevisiae URE2, A39609; S. cerevisiae GSTI, P40582; S. pombe GST3, AAK59430; S. cerevisiae GSTII, Q12390; I. orientalis GSTY1, BAA77459; and I. orientalis GSTY2, S16178. crossover between similar proteins identified by searching with gstA and with the other two A. fumigatus GSTs. GstB and C, however, share similarity with several of the same proteins, although these are less similar than the gstA-like sequences. In addition, both gstB and gstC displayed some similarity with the URE2 group of nitrogen metabolism proteins that have been identified from several Saccharomyces species (Fig. 2), but which have not been shown to exhibit GST activity with CDNB (Rai et al., 2003); this similarity was not evident with gstA. URE2 from $S$. cerevisiae was shown to be involved in defense against heavy metal ions and oxidative stress (Rai et al., 2003). Recently, a putative GST sequence was identified from the A. fumigatus genome as part of a coregulated gene cluster, which is postulated as responsible for production of gliotoxin (Gardiner et al., 2004). While this GST has not been characterised and is not the same as any of the GSTs described here, it suggests a metabolic role for GSTs in A. fumigatus, as has been postulated in other organisms (Hayes and Pulford, 1995), and also suggests there may be further, as yet undiscovered, GSTs in this fungus.

\section{Expression, purification, and activity analysis of recombinant GST proteins}

Protein expression plasmids pPXAgst $A$, pPXAgst $B$, and pPXAgst $C$, consisting of the vector pProEx-Hta containing the open reading frames of gst $A$ (cDNA), gst $B$, and $g_{s t} C$ respectively, were transformed into $E$. coli $\mathrm{DH} 5 \alpha$ and expression induced by the addition of

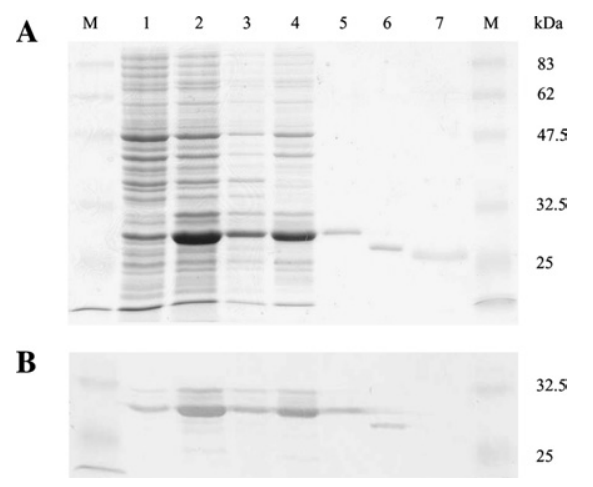

Fig. 3. SDS-PAGE and Western blot analysis of recombinant GST expression in E. coli. Duplicate SDS-PAGE gels were loaded as follows: M, protein size marker; lane 1, uninduced $E$. coli harbouring expression plasmid pPXAgst $C$; lane 2, E. coli harbouring expression plasmid pPXAgst $C$ induced with $0.6 \mathrm{mM}$ IPTG; lane 3 , insoluble proteins extracted from induced $E$. coli; lane 4, soluble proteins extracted from induced $E$. coli; lane 5, (His) 6 -purified recombinant gstC $(5 \mu \mathrm{g})$; lane 6, (His) 6 -purified recombinant gstB $(5 \mu \mathrm{g})$; and lane 7 , (His) ${ }_{6}$-purified recombinant gstA $(4 \mu \mathrm{g})$. One gel was Coomassie stained (A) and the other was probed with anti-(His) ${ }_{6}$ murine monoclonal antibody to identify recombinant proteins (B). GstB was purified in the same manner as gstC. Both gstB and gstC were detected with anti-(His) ${ }_{6}$ monoclonal antibody, but not gstA. However, the identity of this protein was confirmed by mass spectrometry. 
$0.6 \mathrm{mM}$ IPTG (Fig. 3). All three recombinant proteins were present in cell lysate supernatants, indicating solubility, and recombinant gstB and gstC were purified using the $\mathrm{N}$-terminal (His) ${ }_{6}$-tag, with a yield of approximately 17 and $18 \mathrm{mg}$ per gram of $E$. coli cells cultured, respectively (Fig. 3). Detection of recombinant gstA using the (His) 6 tag was not possible, and large-scale metal chelate affinity chromatography was required to purify sufficient enzyme for activity analysis (Fig. 3; Table 2). Purified recombinant proteins were analysed by MALDI-TOF MS and peptides (following tryptic digestion) were identified corresponding to the theoretical amino acid sequence for all three proteins whereby 4/ 83 peptides ( $20 \%$ sequence coverage), $7 / 57$ peptides ( $34 \%$ sequence coverage), and $4 / 46$ peptides ( $15 \%$ sequence coverage) were observed for recombinant gstA, B, and $\mathrm{C}$, respectively. SDS-PAGE data confirm molecular masses of 26, 27, and $30 \mathrm{kDa}$ for gst A, B, and C, respectively (Fig. 3). These are consistent with theoretical molecular masses of 28.99 and $28.72 \mathrm{kDa}$ for the (His) $6_{6}^{-}$ tagged gstB and gstC proteins, but smaller than the theoretical mass of $32.76 \mathrm{kDa}$ for gstA. Protein molecular mass analysis via FPLC gel filtration chromatography (Superose 6) confirmed the dimeric status of purified gstB $(56 \mathrm{kDa})$ and $\mathrm{C}(61 \mathrm{kDa})$, respectively (data not shown). No binding to glutathione-Sepharose affinity columns was observed for any recombinant GST (data not shown). Furthermore, attempts to purify native gstA to $\mathrm{C}$ from $A$. fumigatus extracts by GSH affinity chromatography were unsuccessful (data not shown), which has also been noted in the purification of some theta GST enzymes (Hayes and Pulford, 1995). Crystal structures of some theta GSTs have indicated that the GSH-binding site may be sited further inside the protein than in other classes of GST, and that conventional GSH-affinity matrices may be unable to bind the active site (Hayes and Pulford, 1995).

The enzymatic activities of purified, recombinant gstA-C were assessed with several substrates. CDNB,

Table 2

Specific activities of purified recombinant gstA, gstB, and gstC with both glutathione transferase and glutathione peroxidase substrates

\begin{tabular}{llll}
\hline Substrate & \multicolumn{3}{l}{ Specific activity $\left(\mathrm{U} \mathrm{mg}^{-1}\right)( \pm \mathrm{SD})$} \\
\cline { 2 - 4 } & gstA & gstB & gstC \\
\hline CDNB & $0.004 \pm 0.0001$ & $0.025 \pm 0.0028$ & $0.006 \pm 0.0005$ \\
DCNB & N.D. & N.D. & N.D \\
Ethacrynic acid & N.D. & N.D. & N.D \\
Cumene & $0.019 \pm 0.0009$ & $0.145 \pm 0.0088$ & $0.025 \pm 0.006$ \\
$\quad$ hydroperoxide & & & \\
\hline
\end{tabular}

GST activity was assayed with CDNB, DCNB and ethacrynic acid, and glutathione peroxidase activity was assayed with cumene hydroperoxide. Units are expressed as micromoles substrate utilised per minute. Specific activities stated are the average of five replicates and were corrected against blank reactions that had been performed in triplicate. N.D., no activity detected.
DCNB, and ethacrynic acid are substrates for glutathione transferase activity, whereas cumene hydroperoxide is a substrate for glutathione peroxidase activity (Veal et al., 2002). GstA-C exhibited low level GST and glutathione peroxidase activities, which were detectable due to the high concentrations of recombinant enzyme obtained (Table 2). GstB exhibited a four to six times higher specific activity against $\mathrm{CDNB}$ than either gstA or $\mathrm{C}$, respectively. No activity was observed for either enzyme when DCNB or ethacrynic acid were used as substrates. The specific activity observed against cumene hydroperoxide was almost six times higher for gstB then gstA/C (Table 2). Although relatively lower, the actual ratio of relative activity against CDNB was similar to that previously found (Veal et al., 2002) whereby $S$. pombe gst1 showed three times greater activity towards CDNB than gst2. Conversely, the glutathione peroxidase activity of $S$. pombe gst 2 was about twice that observed for gst 1 albeit at a six times (approx.) lower level to that found for $A$. fumigatus gstB. GST specific activity determinations for $S$. pombe gst1 (Kim et al., 2001) were carried out on cell lysates using impure gst1, thus making exact comparison difficult. However, gst 1 activity was detectable against CDNB with no glutathione peroxidase activity evident. $S$. pombe gst2 also possesses glutathione transferase activity, as measured by CDNB conjugation (Cho et al., 2002). The specific activities of the $A$. fumigatus GSTs are low in comparison with other characterised GSTs, however, any loss of activity due to the presence of the (His) ${ }_{6}$ tag is unlikely as TEV protease removal of the (His) ${ }_{6}$ tag (from gstC) did not result in enhanced activity (data not shown). Ultimately, activity analysis of the native GSTs is required to fully assess activity.

\subsection{Induction of $A$. fumigatus GST expression with $\mathrm{CDNB}$ and $\mathrm{H}_{2} \mathrm{O}_{2}$}

GSTs are thought to be involved in the response against external and cellular toxins, and may also aid the cell when challenged by oxidative stress. After initial growth in the absence of either CDNB or $\mathrm{H}_{2} \mathrm{O}_{2}$, A. fumigatus was further cultured in the presence of $200 \mu \mathrm{M}$ CDNB or $5 \mathrm{mM} \mathrm{H}_{2} \mathrm{O}_{2}$ and expression levels of gst A, gst $B$, and $g s t C$ determined by RT-PCR (Fig. 4). Moreover, the difference in amplicon size for $g s t A$ can be seen thereby confirming the removal of intronic sequence. Primers specific for the calmodulin gene (Romero et al., 2003) were used to confirm absence of genomic DNA and RNA equivalence between time-points. Low basal expression of $g s t A$ and $g_{s t} C$ was detected, but $g_{s t} B$ expression was not detectable under basal conditions. Upon induction with CDNB, all three gst genes were upregulated within one hour, with gst $A$ showing a 10 -fold induction (approximately) and gst $C$ showing at least a 4fold induction at all time-points, and gst $B$ exhibiting only weak induction, with a $20 \%$ drop in RNA expression at 

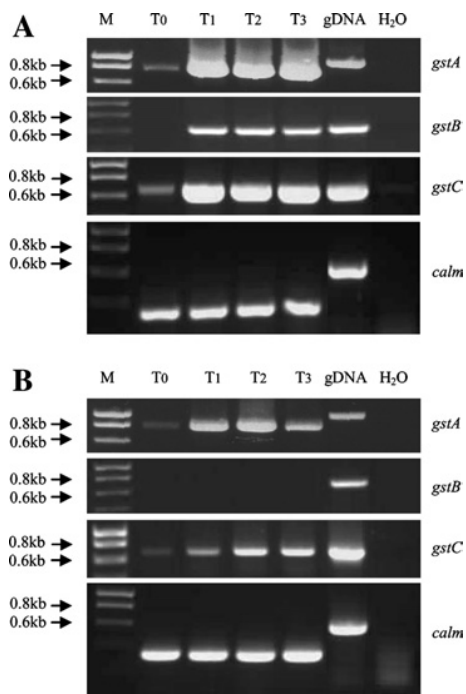

Fig. 4. RT-PCR of A. fumigatus cDNA isolated from cultures induced with either CDNB (A) or $\mathrm{H}_{2} \mathrm{O}_{2}(\mathrm{~B})$. A. fumigatus RNA was isolated prior to induction $\left(T_{0}\right), 1 \mathrm{~h}$ post-induction $\left(T_{1}\right), 2 \mathrm{~h}$ post-induction $\left(T_{2}\right)$, and $3 \mathrm{~h}$ post-induction $\left(T_{3}\right)$. PCR was performed on cDNA using gst $A$, $B$, and $C$ specific primers and primers specific for the calmodulin gene (CALM) (Romero et al., 2003). Optimal cDNA amplification was found to require 45 cycles of PCR. Agarose gels were loaded as follows: M, DNA size marker; $T_{0} ; T_{1} ; T_{2}$; and $T_{3}$; gDNA, genomic DNA control; and $\mathrm{H}_{2} \mathrm{O}$, no DNA control. Expression of all three gst genes was observed when induced with $\mathrm{CDNB}$, and induction of $g s t A$ and gst $C$ only was seen with $\mathrm{H}_{2} \mathrm{O}_{2}$ induction.

$3 \mathrm{~h}$ post induction (Fig. 4A). This result is quite interesting as it is somewhat at variance with the above observation that gstB exhibits greater activity towards CDNB than does gstA or gstC, however it is possible that the higher specific activity of gstB may necessitate production of smaller amounts of actual protein to conjugate available CDNB. Previous work, whereby $\beta$-galactosidase expression was placed under the control of the upstream regulatory regions associated with gst1 and gst2 of $S$. pombe, has shown that gst $1^{+}$gene expression was enhanced by mercuric chloride and menadione (generates superoxide radicals), whereas gst 2 expression was only significantly induced by the presence of $o$-dinitrobenzene (o-DNB) (Cho et al., 2002; Kim et al., 2001; Shin et al., 2002). $o$-DNB also induces GST gene expression in I. orientalis (Tamaki et al., 1999).

In the work presented here, exposure of cells to $\mathrm{H}_{2} \mathrm{O}_{2}$ did not result in gst $B$ induction. However, weak induction of $g s t C$ was detected within $1 \mathrm{~h}$ post-induction (approx. 3-fold increase over $T_{0}$ ) which stabilised at 2 and $3 \mathrm{~h}$ (approx. 7-fold increase over $T_{0}$ ). In addition, strong expression of $g s t A$ at $1 \mathrm{~h}$ post-induction was evident (at least 5-fold increase) which appeared to further increase at $2 \mathrm{~h}$ (approx. 10-fold increase over $T_{0}$ ) before reducing at $3 \mathrm{~h}$ post-induction (approx. 4.5-fold with respect to $T_{0}$ ), possibly due to depletion of added $\mathrm{H}_{2} \mathrm{O}_{2}$ (Fig. 4B). GstB and $g s t C$ were shown to be differentially inducible by $\mathrm{H}_{2} \mathrm{O}_{2}$, whereby up-regulation of $g s t C$ expression only was observed. This indicates the possibility of different roles for these proteins within the organism when subjected to environmental stress. Previous work has also indicated that expression of $g_{s t} 1$, as well as gst2, in $S$. pombe is induced by $\mathrm{H}_{2} \mathrm{O}_{2}$. This observation suggests potentially different mechanisms are involved in the response to oxidative stress for both organisms and that the A. fumigatus gstB plays a different role than gst1 in $S$. pombe - an hypothesis supported by greater sequence divergence between $g s t B$ and $C$ in $A$. fumigatus compared to the corresponding genes in $S$. pombe (Fig. 2). It is intriguing that $g s t B$ expression was not induced in the presence of $\mathrm{H}_{2} \mathrm{O}_{2}$, yet it exhibited the greatest glutathione peroxidase activity against cumene hydroperoxide. In vivo, it is postulated that GST enzymes may be involved in detoxification of secondary oxidation products produced by initial conversion of reactive oxygen species and $\mathrm{H}_{2} \mathrm{O}_{2}$ by enzymes such as glutathione peroxidase and superoxide dismutase (Hayes and McLellan, 1999). These secondary oxidation products include lipid and DNA oxidation products, similar to cumene hydroperoxide, and it is possible that gst $B$ expression is induced by the secondary oxidation products rather than directly by $\mathrm{H}_{2} \mathrm{O}_{2}$. Thus, a putative secondary compound required for induction of gst $B$ may not have been produced when $\mathrm{H}_{2} \mathrm{O}_{2}$ was used as the inducer. A. fumigatus gstA was identified via homology searching of the A. fumigatus genome using $A$. nidulans gstA. In fact, these proteins exhibit a very high degree of sequence similarity $(83 \%)$ which indicates that they may share a common function in both Aspergillus spp. Fraser et al. (2002) have shown that gst $A$ encodes a GST which is involved in xenobiotic and metal ion resistance in A. nidulans. A. fumigatus gst $A$ was strongly up-regulated in the presence of both CDNB and $\mathrm{H}_{2} \mathrm{O}_{2}$. In addition, gst $A$ harbours sequences upstream of the initial ATG with similarity to the xenobiotic (XRE) and antioxidant responsive elements (ARE) involved in regulation of mammalian GSTs (Hayes and Pulford, 1995; Rushmore et al., 1991; Rushmore and Pickett, 1993). Although such responsive elements have not been demonstrated to be functional in the regulation of fungal GSTs to date, XRE and ARE have been identified in the promoter regions of gst genes in S. cerevisiae (Choi et al., 1998). DNA sequences from the A. fumigatus genome (http:// www.tigr.org) $2 \mathrm{~kb}$ upstream of each gst gene were examined for the consensus XRE (TNGCGTG) and ARE (TGACNNNGC) regions. Several XRE and ARE-like regions were observed upstream of all three gst genes. Examination of transcriptional regulation via promoter analysis linked to a reporter gene would be necessary to confirm whether these responsive elements were functional and involved in regulation of gene expression in the presence of CDNB and $\mathrm{H}_{2} \mathrm{O}_{2}$. The expression of all three gst genes in response to organism exposure to CDNB and of gst $A$ and $C$ following exposure of 
A. fumigatus to $\mathrm{H}_{2} \mathrm{O}_{2}$ indicates a high likelihood that GSTs play a role in the response of $A$. fumigatus to both xenobiotic presence and oxidative stress and, as in other fungal species (Veal et al., 2002), may be involved in mediating anti-fungal drug resistance.

\section{Summary}

Three previously unidentified open reading frames encoding glutathione transferases have been identified in A. fumigatus, which are either constitutively expressed under experimental conditions employed ( $g$ st $A$ and $C$ ) or inducible in response to xenobiotic $(g s t A, B$, and $C$ ) or oxidative stress (gst $A$ and $C$ ). Recombinant proteins corresponding to all three genes have been expressed and enzymatic activity characteristic of glutathione transferase and glutathione peroxidase defined. The availability of this information will facilitate further exploration of the possible role(s) played by GST in xenobiotic tolerance and mediating response to oxidative stress in A. fumigatus.

\section{Accession numbers}

Sequences for the three Aspergillus fumigatus glutathione transferases described are deposited in GenBank under Accession Nos. AY770045, AY770043, and AY770044 (gst $A$ to $C$ ) and AY770046 (gst A cDNA).

\section{Acknowledgments}

This work was funded under the IRCSET/Enterprise Ireland Basic Research Grant Programme and the Irish Government Programme for Research in Third Level Institutions. Rachel Geraghty was funded by Dublin City Council studentship scheme, Claire Neville was a recipient of a Daniel O'Connell Fellowship from NUI Maynooth. Preliminary sequence data was obtained from The Institute for Genomic Research website at http://www.tigr.org. Sequencing of A. fumigatus was funded by the National Institute of Allergy and Infectious Disease U01 AI 48830 to David Denning and William Nierman, the Wellcome Trust, and Fondo de Investicagiones Sanitarias.

\section{References}

Bertout, S., Badoc, C., Mallie, M., Giamimis, J., Bastide, J., 2002. Spore diffusate isolated from some strains of Aspergillus fumigatus inhibits phagocytosis by murine alveolar macrophages. FEMS Immunol. Med. Microbiol. 33, 101-106.

Bradford, M., 1976. A rapid and sensitive method for the quantitation of microgram quantities of protein utilizing the principle of protein-dye binding. Anal. Biochem. 72, 248-254.
Cha, C.J., Coles, B.F., Cerniglia, C.E., 2001. Purification and characterization of a glutathione $S$-transferase from the fungus Cunninghamella elegans. FEMS Microbiol. Lett. 203 (2), 257-261.

Cho, Y.W., Park, E.H., Fuchs, J.A., Lim, C.J., 2002. A second stressinducible glutathione S-transferase gene from Schizosaccharomyces pombe. Biochim. Biophys. Acta Gene Struct. Expr. 1574 (3), 399402.

Choi, J.H., Lou, W., Vancura, A., 1998. A novel membrane-bound glutathione $S$-transferase functions in the stationary phase of the yeast Saccharomyces cerevisiae. J. Biol. Chem. 273 (45), 2991529922.

Daly, P., Kavanagh, K., 2001. Pulmonary Aspergillosis: clinical presentation, diagnosis and therapy. Br. J. Biomed. Sci. 58, 197-205.

Denning, D.W., 1998. Invasive aspergillosis. Clin. Inf. Dis. 26, 781-805.

Dowd, C.A., Buckley, C.M., Sheehan, D., 1997. Glutathione $S$-transferases from the white-rot fungus, Phanerochaete chrysosporium. Biochem. J. 324, 243-248.

Dowd, C.A., Sheehan, D., 1999. Variable expression of glutathione $S$ transferase isoenzymes in the fungus, Mucor circinelloides. FEMS Microbiol. Lett. 170 (1), 13-17.

Dower, W.J., Miller, J.F., Ragsdale, C.W., 1988. High-efficiency transformation of Escherichia coli by high-voltage electroporation. Nucleic Acids Res. 16 (13), 6127-6145.

Ellis, D., 2002. Amphotericin B: spectrum and resistance. J. Antimicrob. Chemother. 49, 7-10.

Foley, V., Sheehan, D., 1998. Glutathione $S$-transferases of the yeast Yarrowia lipolytica have unusually large molecular mass. Biochem. J. 333, 839-845.

Fraser, J.A., Davis, M.A., Hynes, M.J., 2002. A gene from Aspergillus nidulans with similarity to URE2 of Saccharomyces cerevisiae encodes a glutathione $S$-transferase which contributes to heavy metal and xenobiotic resistance. Appl. Environ. Microbiol. 68 (6), 2802-2808.

Gardiner, D.M., Cozijnsen, A.J., Wilson, L.M., Pedras, M.S.C., Howlett, B.J., 2004. The sirodesmin biosynthetic gene cluster of the plant pathogenic fungus Leptosphaeria maculans. Mol. Microbiol. 53 (5), $1307-1318$.

Habdous, M., Vincent-Viry, M., Visvikis, S., Siest, G., 2002. Rapid spectrophotometric method for serum glutathione $S$-transferases activity. Clin. Chim. Acta 326 (1-2), 131-142.

Habig, W.H., Jakoby, W.B., 1981. Assays for differentiation of glutathione-S-transferases. Methods Enzymol. 77, 398-405.

Hayes, J.D., McLellan, L.I., 1999. Glutathione and glutathione-dependent enzymes represent a coordinately regulated defence against oxidative stress. Free Radic. Res. 31 (4), 273-300.

Hayes, J.D., Pulford, D.J., 1995. The glutathione $S$-transferase supergene family: regulation of GST and the contribution of the isoenzymes to cancer chemoprotection and drug resistance. Crit. Rev. Biochem. Mol. Biol. 30 (6), 445-600.

Kim, H.G., Park, K.N., Cho, Y.W., Park, E.H., Fuchs, J.A., Lim, C.J., 2001. Characterization and regulation of glutathione $S$-transferase gene from Schizosaccharomyces pombe. Biochim. Biophys. Acta Gene Struct. Expr. 1520 (2), 179-185.

Mitchell, C.G., Slight, J., Donaldson, K., 1997. Diffusible component from the spore surface of the fungus Aspergillus fumigatus which inhibits the macrophage oxidative burst is distinct from gliotoxin and other hyphal toxins. Thorax 52, 796-801.

Muryama, T., Amitani, R., Ikegami, Y., Awada, R., Lee, W.J., Kuze, F., 1996. Suppressive effects of Aspergillus fumigatus culture filtrates on human alveolar macrophages and polymorphonuclear leucocytes. Eur. Respir. J. 9, 293-300.

Nicholson, T.P., Rudd, B.A.M., Dawson, M., Lazarus, C.M., Simpson, T.J., Cox, R.J., 2001. Design and utility of oligonucleotide gene probes for fungal polyketide synthases. Chem. Biol. 8, 157-178.

Page, R.D.M., 1996. TREEVIEW: an application to display phylogenetic trees on personal computers. Comput. Appl. Biosci. 12, $357-$ 358. 
Rai, R., Tate, J.J., Cooper, T.G., 2003. Ure2, a prion precursor with homology to glutathione $S$-transferase, protects Saccharomyces cerevisiae cells from heavy metal ion and oxidant toxicity. J. Biol. Chem. 278, 12826-12833.

Romero, B., Turner, G., Olivas, I., Laborda, F., De Lucas, J.R., 2003. The Aspergillus nidulans alcA promoter drives tightly regulated conditional gene expression in Aspergillus fumigatus permitting validation of essential genes in this human pathogen. Fungal Genet. Biol. 40 (2), 103-114.

Rushmore, T.H., Morton, M.R., Pickett, C.B., 1991. The antioxidant responsive element-activation by oxidative stress and identification of the DNA consensus sequence required for functional activity. J. Biol. Chem. 266 (18), 11632-11639.

Rushmore, T.H., Pickett, C.B., 1993. Glutathione $S$-transferases, structure, regulation, and therapeutic implications. J. Biol. Chem. 268 (16), 11475-11478.

Sheehan, D., Meade, G., Foley, V.M., Dowd, C.A., 2001. Structure, function and evolution of glutathione transferases: implications for classification of non-mammalian members of an ancient enzyme superfamily. Biochem. J. 360, 1-16.
Shin, Y.H., Park, E.H., Fuchs, J.A., Lim, C.J., 2002. Characterization, expression and regulation of a third gene encoding glutathione S-transferase from the fission yeast. Biochim. Biophys. Acta Gene Struct. Expr. 1577 (1), 164-170.

Takada, Y., Uda, K., Kawamura, K., Matsuoka, T., 2004. Molecular cloning and characterization of a novel glutathione $S$-transferase gene induced by light stimulation in the protozoan Blepharisma japonicum. FEMS Microbiol. Lett. 231 (2), 185-189.

Tamaki, H., Yamamoto, K., Kumagai, H., 1999. Expression of two glutathione $S$-transferase genes in the yeast Issatchenkia orientalis is induced by $o$-dinitrobenzene during cell growth arrest. J. Bacteriol. 181 (9), 2958-2962.

Thompson, J.D., Higgins, D.G., Gibson, T.J., 1994. CLUSTAL W: improving the sensitivity of progressive multiple sequence alignment through sequence weighting, position-specific gap penalties and weight matrix choice. Nucleic Acids Res. 22 (22), 4673-4680.

Veal, E.A., Toone, W.M., Jones, N., Morgan, B.A., 2002. Distinct roles for glutathione $S$-transferases in the oxidative stress response in Schizosaccharomyces pombe. J. Biol. Chem. 277 (38), 35523-35531. 